"Aristócratas" o "diplomados". Las tensiones en la formación docente de los primeros psicólogos de la Universidad de Buenos Aires

Artículo de Fernando Cazas

Educación, Lenguaje y Sociedad EISSN 2545-7667 Vol. XIV Nº 14 (Abril 2017) pp. 1-17

DOI:http://dx.doi.org/10.19137/els-2017-141403

Fecha de recepción: 07/02/2016

Fecha de aceptación: 09/11/2016

Palabras clave: posición profesional, enseñanza, formación de docentes, psicólogos

Keywords: professional position, teaching, teacher training, psychologists
"Aristócratas" o "diplomados". Las tensiones en la formación docente de los primeros psicólogos de la Universidad de Buenos Aires

\section{"Aristocrats" or "graduates". Tensions in teacher training of the first psychologists at the University of Buenos Aires}

\author{
Fernando Cazas \\ Universidad de Buenos Aires, Argentina \\ fernandocazas@yahoo.com.ar
}

\section{Resumen}

Durante los primeros años de la década del 60, egresaron los primeros psicólogos de la carrera de Licenciatura de la Universidad de Buenos Aires [UBA]. Su inserción laboral se inclinó fuertemente hacia el ámbito de la salud. No obstante, muchos de ellos también incursionaron en el ejercicio de la docencia (en especial en cátedras de la propia universidad). A pesar de esto, pocos elegían la carrera de profesorado que se dictaba en la misma facultad de Filosofía y Letras. La necesidad de una formación específica para enseñar, no parece tener lugar entre aquellos primeros psicólogos. Este trabajo se propone analizar algunas de las tensiones puestas en juego en la formación docente de los psicólogos. ¿Qué características tenía la formación docente que ofrecía la UBA a estos? ¿Por qué está formación resultaba poco atractiva? Para el análisis de estos interrogantes se propone trabajar con la categoría de posición docente, en particular con las variantes que proponen Birgin y Pineau 
(2014). Finalmente, se analizan algunas cuestiones de la formación docente hoy, a la luz de los análisis realizados sobre el caso de los primeros psicólogos.

During the early years of the $60 \mathrm{~s}$, the first psychologists graduated from the Bachelor of UBA. The employment of these leaned strongly toward the field of health. However, many also raided the practice of teaching (especially chairs of the university). Despite this, few chose the career of teachers who are dictated in the same faculty. The need for specific training for teaching, does not seem to occur between those early psychologists. This paper analyzes some of the tensions at play in teacher training for those psychologists. What characteristics did the teacher training offered by the UBA to these? Why is Training was unattractive? For the analysis of these questions it intends to work with the category of teaching position, particularly those describing Birgin and Pineau (2014). Finally, some issues of teacher education are discussed today, in light of the analyzes performed on the case of the first psychologists.

\section{Introducción}

Z1 presente trabajo se propone analizar el lugar de la formación Cdocente en la Universidad de Buenos Aires en los años '60. Para ello, se centra la atención en el caso de los primeros graduados de la carrera de psicología que, como parte de su ejercicio profesional, incursionaron en la enseñanza. Para estos el profesorado podía cursarse en la Facultad de Filosofía y Letras.

Estas primeras cohortes de graduados de la Licenciatura en Psicología tenían la posibilidad de cursar la carrera de Profesorado, ya que ésta estaba contemplada en el flamante plan de estudios. Sin embargo, se observa poco interés por realizar esta carrera de formación docente. 
Podría pensarse que el campo de la educación o el de la enseñanza, no eran de interés para los graduados de una carrera con fuerte inclinación hacia el área de la salud. No obstante, existen numerosos documentos y testimonios que demuestran lo contrario. Muchos de aquellos primeros graduados ejercieron la docencia, en particular, la universitaria. Además, participaron de diversos programas de la Secretaria de Extensión Universitaria vinculados al campo educativo.

Con este cuadro de situación, se propone un análisis desde la categoría de posición docente, en particular desde las posiciones docentes postuladas por Birgin y Pineau (2014). Este análisis permite avanzar en la comprensión de porqué la carrera de profesorado no resultaba atractiva para los primeros graduados de la licenciatura en psicología, aun cuando el campo de la educación y de la enseñanza, sí lo era.

Finalmente, este análisis permite pensar en algunos debates actuales sobre la formación docente. En particular en lo que refiere a la formación de profesores para el nivel secundario y de educación superior.

\section{Los primeros psicólogos y la enseñanza}

Cuando se crea la carrera de psicología en la UBA en 1957, el plan de estudios aprobado por el Consejo Superior ${ }^{1}$, contemplaba el título de licenciado, de profesor y de doctor. Rápidamente, la carrera de licenciado alcanza una matrícula numerosa en comparación con otras carreras de la Facultad de Filosofía y Letras.

La carrera de licenciado y su posterior inserción laboral (en la clínica) eran muy bien ponderadas por los alumnos, en cambio la carrera de profesorado era casi ignorada por la misma población. Resulta llamativo, entonces, que muchos de los primeros graduados de la licenciatura eligieran la docencia universitaria, que en algunos casos, llegó a extenderse por más de 20 años. Ahora bien, ante la consulta, los docentes aseguran que no sería necesaria la formación específica.

A continuación, algunos fragmentos de testimonios ${ }^{2}$ de graduados y ex estudiantes, en esta línea: 
P: Y en esa época, sus compañeros de la licenciatura... ¿se hablaba de esta opción del profesorado?

R: No, no... ¿sabes lo que pasa? Yo no sé si sigue pasando ahora... es el que se recibía ¡pum! caía en el consultorio. Como el modelo médico. El médico que se recibe ¿qué idea tiene? El 98\% van al consultorio y los otros van a investigación o a otras cosas, pero el modelo médico era el que predominaba, así que, por ahí no les interesaba la docencia, pero curiosamente pensaban que la docencia se ejercía sin necesidad de tener ningún atributo en especial, sin tener elementos de didáctica o de pedagogía, que el que sabe una disciplina, sabe enseñarla.

P: ¿Y cómo se enteró usted que la carrera ofrecía esa opción del profesorado... al ingresar, había alguna gacetilla, o usted preguntó?

R: Eso no lo recuerdo,... pero uno como siempre estaba digamos más o menos pendiente con los compañeros y en el Departamento de Psicología había algunos docentes que eran compañeros también ${ }^{3}$ (entrevista, Setiembre 2013).

De este modo, la práctica clínica, aparecía casi como una opción única para el egresado de la carrera de Licenciatura. La docencia pareciera no ocupar un lugar preponderante a la hora de formarse, pero sí es una opción con respecto a la inserción laboral. En este caso ella se daba mayormente en las propias cátedras de la Licenciatura. En estos casos, la ausencia de una formación docente no constituía un obstáculo para la inclusión de los psicólogos. La formación para enseñar no parece ser relevante para las cátedras de la Licenciatura, tal como se manifiesta en el siguiente testimonio:

(...) no tengo un solo recuerdo de habernos transmitido algún concepto de pedagogía o alguna instrucción, alguna orientación al respecto, se suponía que si sabíamos la materia, teníamos que saber enseñar. Era un presupuesto bastante generalizado en todas las cátedras, no en esa cátedra en particular ${ }^{4}$ (entrevista, Abril 2012). 
Por otra parte, la necesidad de la formación específica para la enseñanza, tampoco era considerada en otros espacios de capacitación y actualización que proponían cursos y seminarios valorados para los egresados de la carrera de Psicología. Es posible observar, desde una mirada corporativa, en planteos como el de quien fuera uno de los presidentes de la Asociación de Psicólogos de Buenos Aires:

(...) y allí tampoco a nadie se le ocurría, estaba ausente en el imaginario de los psicólogos hacer algún curso, alguna formación complementaria para ejercer la docencia ${ }^{5}$ (entrevista, Abril 2012).

Resulta también pertinente mencionar la dificultad para ubicar recuerdos de los docentes o de las clases en los testimonios de quienes cursaron la carrera de Profesorado, si lo comparamos con los recuerdos de la carrera de Licenciatura. En estos últimos, abundan nombres de profesores y recuerdos sobre los recursos didácticos que ellos utilizaban en sus clases. Incluso, algunos entrevistados reconocen haberlos imitado, en sus inicios como docentes.

Esto puede observarse en varios testimonios, como en el siguiente ejemplo:

P: De la cursada del profesorado, recuerda alguna experiencia significativa o algún docente...que lo haya sorprendido por ahí, con alguna propuesta de trabajo, algo que saliera de lo común....

R: No, no. Absolutamente. Para nada...

P: ¿Recuerda el nombre de algún docente?

R: No. Y eso... es significativo... A parte de la edad, que a uno se le borran tantas cosas...

P: Bueno, no, pero seguro que si yo le pregunto por algún docente de la licenciatura seguramente alguno aparece...

R: Nooo, si, si, de la licenciatura $\mathrm{si}^{6}$ (entrevista, Setiembre 2013).

Lo mismo sucede al momento de recordar compañeros de cursada: 
P: ¿Recuerda algún compañero de la licenciatura que haya hecho el profesorado, que haya compartido con Ud. una materia?

R: No... recuerdo, no (entrevista, Setiembre 2013).

Otra cuestión que resulta relevante en esta línea de indagación, es la cantidad de egresados de la carrera de Licenciatura en comparación con la de Profesorado. Los egresados de este último representan aproximadamente el 2,5\% del total de egresados de la Licenciatura. Se ha podido ratificar, a través de documentación existente, que la cantidad de egresados de la carrera de profesorado era poco significativa en relación con la cantidad de egresados que tenía la Licenciatura ${ }^{8}$. Pero además, se ha podido corroborar con testimonios que van en la misma línea:

$\mathrm{P}:$ ¿Y recordás si dentro de la carrera de psicología, cómo se valoraba el tema del profesorado?, ¿O en general se lo consideraba una carrera menor, hacer el profesorado?

$\mathrm{R}$ : La gente que estudió conmigo, que se recibió conmigo de licenciada, no estudiaban profesorado. En mi caso, como yo ya estaba orientada hacia la docencia, porque ya trabajaba en escuelas, y toda mi idea era seguir la docencia, porque a mí siempre me gustó enseñar... Para mí era una salida necesaria, y no lo había dudado, de hacerla. Pero no es que mis compañeros lo iban a hacer. No, no éramos muchos ${ }^{9}$ (entrevista, Agosto 2013).

Con lo hasta aquí expuesto, no sería desatinado hablar de una especie de "desdibujamiento" de la carrera de Profesorado con relación a la carrera de Licenciatura. En este sentido, los testimonios de los graduados de las primeras cohortes (entre 1963 y 1970 aproximadamente), muestran a la formación docente en un lugar secundario en relación con la licenciatura. El saber pedagógico y didáctico aparece opacado por el saber disciplinar. ¿Tendrá esto que ver con un desinterés por el campo de la educación y la enseñanza, por parte de estos graduados en Psicología? ¿Será que la inserción en el campo de la salud y de la práctica clínica eclipsaba cualquier otra opción profesional? 


\section{Educación y enseñanza}

Existen evidencias de que el ejercicio de la docencia no resulta ajeno a los intereses de los primeros psicólogos egresados de la UBA. Como ya se dijo, muchos de los integrantes de esas primeras cohortes de graduados ejercieron la docencia universitaria durante muchos años (Diamant, 2010). El testimonio que sigue da cuenta de la inserción en la docencia universitaria de los primeros graduados en Psicología:

(Tras las renuncias de 1966) “...acceden los psicólogos a ser profesores. Que hasta ese día no eran más que auxiliares docentes los psicólogos. Profesores eran o psicoanalistas o gente venida de Filosofia, como Berstein; pero no eran psicólogos. Cuando se acepta la invitación de Ipar, por ejemplo en lugar de Ulloa en Psicología Institucional viene Malfé. En lugar de Berstein en Técnicas Proyectivas viene Pichona Ocampo, como titular. No sé si lo nombraban Titular o Profesor Adjunto a Cargo pero la cuestión es que está a cargo de la materia. Es en ese momento que los psicólogos pasan a estar a cargo de materias. Hasta entonces eran de Jefes de Trabajos Prácticos para abajo, ni Adjuntos" (Diamant, 2010, p. 114).

El campo de la educación, tampoco fue ajeno al interés de los primeros psicólogos. Una interesante experiencia para analizar esta cuestión, es el Programa para el desarrollo integral de Isla Maciel. Este programa dependía de la Dirección de Extensión Universitaria de la UBA (Urrutia, 2009; Diamant y Urrutia, 2013). De este programa participaron muchos de los alumnos de la licenciatura. El programa incluía un Centro Juvenil que estaba coordinado por una licenciada en Psicología y que desarrollaba tareas educativas extraescolares (Urrutia, 2009). En ese Centro participaban alumnos de varias carreras, entre ellas los de la Licenciatura en Psicología.

Esta actividad en Isla Maciel, es recordaba con entusiasmo por los graduados que participaron. El siguiente testimonio da cuenta de ello: 
...la vida en la Facultad era muy intensa, el estudio, las materias, los trabajos para Extensión. Me acuerdo haber colaborado con los trabajos que se hacian en Isla Maciel, haber colaborado en investigaciones, o en encuestas, para libros también" (Diamant, 2010, p. 240).

"Muchos de nosotros trabajábamos en cosa asistencial-de 'pinches', no? por supuesto- o en cosas hospitalarias, pero no necesariamente solo psicoterapéuticas. De un principio yo me acuerdo, yo fui varios años a Isla Maciel, ayudando de 'pinche', a estimular actitudes hacia el aprendizaje, hacia la participación grupal, hacia...etc.etc... (Diamant, 2010, p. 103).

La actividad que los estudiantes de la carrera de licenciatura realizaban en Isla Maciel, incluía al campo de la educación además del campo de la salud mental.

Lo mismo sucede con la participación de primeros psicólogos en actividades del flamante Departamento de Orientación Vocacional de la UBA. Aquí también es clara la vinculación con el campo de la educación y de lo escolar (Diamant, 2010; Buchbinder, 1997; Borinsky, 1998).

En principio, los elementos de los que se dispone hasta aquí, no parecen avalar la idea de que el desinterés por la carrera de Profesorado obedeciera a un desinterés por el campo educativo por parte de los estudiantes de la Licenciatura.

\section{Aprender a enseñar}

Si estos primeros psicólogos, interesados por lo educativo y por la enseñanza de la psicología, no elegían formarse para enseñar en la carrera de profesorado ¿cómo aprendieron a enseñar? Aquí resulta pertinente pensar en la categoría de saberes tácitos que trabaja Terigi (2012) entre otros. Podría pensarse que estos primeros graduados en psicología que ejercieron la docencia no recibieron una formación docente "explícita" 
pero sí una formación en términos de saberes tácitos. ¿De quiénes recibieron esa formación no explícita? Una posibilidad es pensar que la recibieron de sus propios docentes de la Licenciatura. El primer cuerpo de profesores de la carrera de Licenciatura no estaba, lógicamente, formada por psicólogos. Estaba conformada por un conjunto de profesionales destacados provenientes de otras disciplinas (filosofía, medicina, psicoanálisis). Algunos de estos primeros docentes son reconocidos por los ex estudiantes como verdaderos maestros ${ }^{10}$. De estos docentes, los estudiantes van a aprender a enseñar. El ejemplo paradigmático será José Bleger, quien aparece en numerosos testimonios como verdadero modelo a seguir a la hora de ejercer la docencia. En Bleger convergen las características más estimadas por los estudiantes de aquellas cohortes de los años '60: es un prestigioso psicoanalista con gran dominio disciplinar y una amplia cultura general, una persona comprometida con lo social y adscribe políticamente a las ideas de izquierda.

No es difícil rastrear este tipo de ideales en determinado sector social de clase media urbana en la Argentina de los 60. La preocupación por lo social y la necesidad de implicarse activamente como universitarios en el cambio social, son una marca de época en la vida universitaria de la UBA. Por otro lado, el psicoanálisis en ese momento, se mostraba como algo novedoso y que prometía ser eficaz para alcanzar ese propósito (Diamant, 2010; Plotkin, 2003).

De esta manera, podría pensarse que estos primeros psicólogos entendieron que la forma de enseñar era imitar a sus docentes modelos, estos maestros que despertaban su admiración. Cabe destacar que estos docentes carecían de un título formal de profesores. Su inserción como docentes en la carrera de Licenciatura obedecía a su gran prestigio profesional y a sus condiciones personales.

\section{Un análisis desde la categoría de posición docente}

Si bien, en trabajos anteriores (Cazas y Diamant, 2014), se han barajado diversas hipótesis sobre el motivo de este opacamiento de la carrera de profesorado en el caso de los licenciados en psicología de 
los '60, el análisis de la cuestión desde la categoría de posición docente puede sumar a una mejor comprensión del tema.

Myriam Southwell (2014) describe la categoría en los siguientes términos:

... se compone de la circulación de sentidos y de los discursos que regulan y organizan el trabajo de enseñar, y se refiere específicamente a los múltiples modos en que los sujetos enseñantes asumen, viven y piensan su tarea, y los problemas, desafíos y utopías que se plantean en torno a ella (p. 166).

La posición docente es una forma de mirar las identidades docentes que se construyen en el contexto del ejercicio profesional pensadas como una relación. Y esta relación es el producto de un conjunto de cuestiones puestas en juego, no solo del presente sino también del pasado. La posición no se construye sobre el contexto inmediato pero también sobre las herencias y las tradiciones.

Alejandra Birgin y Pablo Pineau (2014) proponen cuatro posiciones docentes para pensar la formación de profesores de secundaria en la Argentina. En orden cronológico son: la aristocracia profesoral; los profesores diplomados; el profesor como profesional de la sociedad salarial; y los profesores ante los efectos de la fragmentación educativa.

Para el presente trabajo interesa rescatar, en particular, dos de estas posiciones: la aristocracia profesoral y los profesores diplomados. Los autores, al referirse a la primera de las posiciones, destacan que:

...el ejercicio de la función de profesor de escuela media no exigía acreditación alguna, sino que estaba mas ligada al capital en estado incorporado, generalmente heredado, al que se accedía por ser parte de la clase política dirigente, haber alcanzado cierto reconocimiento intelectual y en algunos casos poseer un título universitario" (...)"Constituían entonces una "aristocracia" de herencia, que provocaba la admiración de sus alumnos y la identificación con sus pares (Birgin y Pineau, 2014, p. 53 ). 
En relación a la segunda posición mencionada, la de los profesores diplomados, los autores refieren que esta posición se vincula con la aparición de profesores que lo eran por haber obtenido un título en una institución de formación docente que los habilitaba para ejercer como tales. Aquí no se trata de prestigiosos intelectuales, sino de sujetos provenientes de una ascendiente clase media urbana. Dicen Birgin y Pineau:

\footnotetext{
En esta posición, y en forma tendencial, los profesores de secundaria pierden su condición de 'intelectuales orgánicos' de la alta cultura para volverse sus 'intelectuales subordinados', en tanto difusores de dicha cultura (Birgin y Pineau, 2014, p. 57).
}

Si se piensa en términos de estas dos posiciones el caso de la formación docente de los primeros psicólogos de la UBA, podría analizarse como una tensión entre ellas ${ }^{11}$.

En este sentido, los docentes admirados y tomados como modelo (los que se han denominado maestros), pertenecían a una aristocracia profesoral en los términos arriba descriptos. Hombres elegidos para la función docente por su prestigio personal y por su herencia cultural. El ejemplo de Bleger es más que ilustrativo. Los estudiantes de aquella época eligieron a personas como Bleger para imitar a la hora de ser docentes. Ellos también querían pertenecer a esa aristocracia profesoral. Sin embargo, para la década del ' 60 , esta posición ya había dejado paso a la de los "profesores diplomados". Esta era la opción que les ofrecía la carrera de profesorado. Pero, atento a las cuestiones de época que ya se mencionaron, no resulta impropio pensar que esos primeros graduados no sentían ningún interés por acceder a esa posición. Seguramente, algo de lo referido a la autorización cultural (Bhabha, 2002) también se jugaba en la construcción de una posición u otra. En síntesis, los primeros psicólogos querían acceder a la aristocracia profesoral, pero la carrera de profesorado les ofrecía ser diplomados. Negarles la posición de la aristocracia profesoral era negarles la posibilidad de ser como esos 
intelectuales orgánicos que tanto admiraban (ellos y su contexto social), y a cambio les ofrecían ser unos intelectuales subordinados. Esta situación podría pensarse como una tensión entre las dos posiciones docentes. De modo que la formación docente de aquellos primeros psicólogos quedó atrapada entre estas dos lógicas de posiciones.

Por otra parte, el contexto de época también debió haber influido en la generación de esta tensión. La década del ' 60 , marcó a las políticas de formación docente en la Argentina. No obstante, su complejidad amerita un tratamiento aparte.

\section{La formación docente en los '60}

Pablo Pineau, en el libro El principio del fin (2006), plantea un interesante cuadro de situación del campo de la educación entre las décadas de los '50 y '70.

Pineau explica que durante ese período, pueden encontrarse a sectores que sostenían que la pedagogía debía modernizarse. Esto significaba abandonar las corrientes escolanovacionistas y espiritualistas de décadas anteriores, y dar paso a un discurso de modernización tecnocrática.

El discurso pedagógico fue invadido por una visión proveniente de la economía. La teoría del capital humano ponía a la escuela al servicio del proyecto productivo nacional (Birgin, 2007). De esta manera fue como aparecieron en el campo educativo términos como "tasa de retorno", "capital humano", "eficiencia". Todo un grupo de nuevos expertos en educación, que en muchos casos habían sido formados en universidades estadounidenses y los distintos gobiernos del período les dieron importantes lugares en los niveles de decisión del sistema educativo argentino. Agrega Pineau (2006, p. 127) "Toda esta renovación implicó la aparición de un sujeto pedagógico asocial, individualista, racional y planificador".

Este cambio de paradigma impactó también en las prácticas escolares cotidianas. La irrupción de una didáctica de corte neoconductista se impuso como "lo nuevo" "lo moderno" en contraposición con otras posturas didácticas. Los docentes debían adquirir habilidades técnicas 
para realizar su trabajo y debían abandonar las tareas de planificación en manos de los nuevos "técnicos" de la educación (Birgin, 2007).

Para Pineau, este nuevo paradigma alcanzó su momento máximo durante la dictadura del General Onganía, quien no escatimó cuotas de autoritarismo y represión para lograr la modernización de la educación argentina.

Evidentemente, en este contexto, la opción por una carrera de profesorado cuyos egresados tendían rápidamente a incorporarse a una nueva posición docente (la de los profesores como profesionales de la sociedad salarial), se ubicaba en las antípodas de las aspiraciones de los graduados de la licenciatura en psicología. Ellos tenían claro un modelo de mundo nuevo pero lo escolar parecía apuntar a perpetuar el viejo mundo (Simons y Masschelein, 2014). Muy probablemente este contexto de época colaboró en la conformación de esta tensión entre las dos posiciones docentes ya descriptas.

\section{Algunas cuestiones para pensar la formación docente hoy}

Podría pensarse que en aquella pretensión de esos primeros graduados de psicología en la UBA, de querer pertenecer a una aristocracia profesoral, estaba en juego algo del orden de la siguiente pregunta: ¿cualquiera puede ser docente? ¿Cualquiera puede ser autorizado a enseñar? La posición de la aristocracia profesoral se construía sobre la base de una herencia cultural y social. De este modo, Bleger era tomado como modelo no solo por sus virtudes sino también por su herencia cultural. Este requisito, claramente aristocrático, limita el acceso a la condición de profesores a unos pocos elegidos.

Si como afirman Birgin y Pineau (2014), seguramente hoy se esté construyendo una nueva posición docente, está será heredera de las cuatro posiciones docentes descriptas. Esta nueva posición se construirá sobre la base de los debates, las tensiones y los conflictos de las posiciones que la precedieron. La posición docente de hoy se ubica en una línea genealógica en el sentido que lo plantea Hassoun (1996). No se trata de una herencia en sentido de una copia actual del pasado, sino de un 
parecido de familia que conserva en el presente al pasado resignificado. Si se acepta esto, la actual posición docente en construcción conservará algo de aquella aristocracia profesoral. Y esto hoy interfiere a la hora de discutir las políticas de formación docente en las instituciones formadoras. En un momento de claras políticas docentes inclusivas, pareciera que la vieja aristocracia profesoral aún se defiende y acciona desde el pasado conservada en una nueva posición. Quizás se defiende tendiendo algunas trampas como cuando Ezcurra (2011, p. 62) dice que el acceso de amplios sectores sociales a la educación superior, es en verdad una "puerta giratoria" que facilita el ingreso de sectores sociales (excluidos de la aristocracia profesoral), pero permite su rápido egreso por la vía de la deserción y el desgranamiento. O quizás se defienda tendiendo la trampa de la segmentación (Braslavky, 1985) y de la fragmentación (Tiramonti, 2011), permitiendo que sujetos provenientes de sectores sociales no incluidos en una aristocracia profesoral, obtengan su título de profesores pero en instituciones que no califican para que sus egresados accedan a la vieja posición aristocrática.

A la hora de discutir nuevas políticas inclusivas que permitan el acceso a la formación de profesores de distintos sectores sociales, no debería caerse en la ingenuidad de pensar que las cuatro posiciones docentes descriptas por Birgin y Pineau (2014), pertenecen al pasado y no han dejado huella en los docentes actuales. Ese deseo de pertenecer a una aristocracia profesoral aún sigue vivo en muchos estudiantes y docentes de profesorado. Parte de esa tensión que caracterizó a la formación docente de los primeros graduados de psicología en la UBA, aun produce efectos.

\section{Referencias bibliográficas}

Bhabha, H. (2002). El lugar de la cultura. Buenos Aires. Ediciones Manatial.

Birgin, A. y Pineau, P. (2014). Posiciones docentes del profesorado para la enseñanza secundaria en la Argentina: una mirada histórica para pensar el presente. Revista Teoria e Prática da Educação - "Dossier História da 
formação docente: olhares latino-americanos", 18, (1), 47-61.

Birgin, A. (2007). Pensar la formación de los docentes de nuestro tiempo. En Terigi, F. (comp) Diez miradas sobre la escuela primaria (283-285). Buenos Aires: Fundación OSDE/Siglo XXI. .

Borinsky, M. (1998). Las Primeras Estrategias de Inserción Profesional de los Psicólogos. Un corte sincrónico en el estudio de la conformación de un campo profesional. Recuperado de: http://www.elseminario.com.ar/biblioteca/Borinsky_Primeras_estrategias_psicologos.htmel 18 de julio de 2015.

Braslavsky, C. (1985). La discriminación educativa en Argentina. Buenos Aires: Miño y Dávila.

Buchbinder, P. (1997). Historia de la Facultad de Filosofia y Letras. Universidad de Buenos Aires. Buenos Aires: EUDEBA.

Cazas y Diamant, A. (Abril, 2014). Itinerarios borrosos en la formación de profesores en Psicología en la UBA (1965 -1976). Ponencia publicada en Memorias del VIII Congreso Iberoamericano de Docencia Universitaria $y$ de Nivel Superior. Universidad Nacional de Rosario/AIDU.

Davini, M.C. (1995). La formación docente en cuestión: política y pedagogía. Buenos Aires: Paidós.

Diamant, A. (2010). Testimonios de enseñar y aprender: ser psicólogo en la UBA de los 60. Buenos Aires: Teseo.

Diamant, A \& Urrutia, J.P. (2013). Programas Educativos en el Centro de Desarrollo Integral de Isla Maciel: Voces que visibilizan una experiencia de extensión universitaria en la UBA de los '60 Anuario de Historia de la Educación, 14, (2), (p. 42)

Ezcurra, A. (2011). Masificación y enseñanza superior: una inclusión excluyente. Algunas hipótesis y conceptos clave. En Fernández Lamarra, N. y Costa De Paula, M. (comps.) La democratización de la educación superior en América Latina. Límites y Posibilidades. (p. 63) Sáenz Peña: EDUNTREF. 
Hassoun, J. (1996). Los contrabandistas de la memoria. Buenos Aires: Ediciones de la Flor.

Molinari, A. (2005). De cursos y de formaciones docentes. Buenos Aires: Departamento de publicaciones de la Facultad de Derecho de la UBA.

Pineau, P., Mariño, M., Arata, N. y Mercado, B. (2006). El principio del fin. Políticas y memorias de la educación en la última dictadura militar (1976 - 1983). Buenos Aires: Colihue.

Plotkin, M. (2003). Freud en Las Pampas. Buenos Aires: Sudamericana.

Simons, M. y Masschelein, J. (2014). Defensa de la escuela. Una cuestión pública. Buenos Aires. Miño y Dávila.

Southwell, M. y Vassiliades, A. (2014). El concepto de posición docente: notas conceptuales y metodológicas. Revista Educación, Lenguaje y Sociedad, $X I, 163-187$.

Terigi, F (2012). Los saberes de los docentes: formación, elaboración en la experiencia e investigación: documento básico. Buenos Aires: Santillana.

Tiramonti, G. y Montes, N. (Comp.) (2011). La escuela media en debate. Problemas actuales y perspectivas de la investigación. Buenos Aires: FLACSO y Ediciones Manantial.

Urrutia, J. (Noviembre, 2009). Educación popular y el lugar de los Psicólogos. Experiencia en Isla Maciel (1956-1966). Ponencia publicada en Memorias de las XV Jornadas de Investigación en Psicología. Facultad de Psicología-UBA.

Resolución 528 del Consejo Superior del 14/3/57.

\section{Notas}

1 No debe descartarse aquí la fuerte influencia de la tradición academicista de la UBA. Seguramente esta idea de dominio de la disciplina (Davini, 1995; Molinari, 2005), alimentó esta tensión que para enseñar alcanza con el buen entre las posiciones. 
2 Este artículo se ha elaborado a partir de los proyectos de investigación realizados por el Equipo que dirige la Dra. Ana Diamant en la Facultad de Psicología de la UBA, del cual forma parte el autor del artículo. En particular se han tomado en cuenta testimonios e hipótesis presentes en los siguientes proyectos: "Registros didácticos. Itinerarios y trazas socio históricas en la enseñanza de la Psicología para la formación docente. Las marcas en los profesores de Psicología egresados en la UBA (1965-1975)". (UBACyT / Programación 2011-2014) y "La traza didáctica. Recuperación de huellas de maestros en la formación de los primeros Psicólogos en la UBA (19571966)". (UBACyT / Programación 2008-2011). Los testimonios citados fueron recogidos en el marco de estas investigaciones y en el de la tesis doctoral de la Dra. Ana Diamant.

3 Egresado de las carreras de Licenciatura y Profesorado en Psicología. Entrevista realizada en Setiembre de 2013, en Buenos Aires por Cazas, F.

4 Egresado de la carreras de Licenciatura en Psicología. Entrevista realizada en Abril de 2012, en Buenos Aires por Fernando Cazas y Nora Salles.

5 Egresado de la carreras de Licenciatura en Psicología. Entrevista realizada en
Abril de 2012, en Buenos Aires por Fernando Cazas y Nora Salles.

6 Egresado de las carreras de Licenciatura y Profesorado en Psicología. Entrevista realizada en Setiembre de 2013, en Buenos Aires por Cazas, F.

7 Egresado de las carreras de Licenciatura y Profesorado en Psicología. Entrevista realizada en Setiembre de 2013, en Buenos Aires por Cazas, F.

8 Fuente http://www.uba.ar/institucional/ censos/series/cuadro9.htm. Última entrada 20/09/2015.

9 Egresada de las carreras de Licenciatura y Profesorado en Psicología. Entrevista realizada en Agosto de 2013, en Buenos Aires por Cazas, F.

10 Aquí la expresión "maestro" no se utiliza en el sentido vulgar sino en la acepción de aquel que más sabe en su oficio.

$11 \mathrm{Si}$ bien las posiciones enunciadas por los autores refieren solo a los profesores de nivel secundario, bien pueden ser aplicadas a la Universidad de Buenos Aires atento a la íntima ligazón que existió desde los orígenes entre ambos niveles educativos. 